\title{
Der eigene Schmerz und der Schmerz der anderen
}

Versuch über die epistemische Dimension der Verletzlichkeit

Fabian Bernhardt

Am 8. November 1903 schrieb Franz Kafka, damals 20-jährig, einen Brief an seinen Freund Oskar Pollak. »Wenn Du vor mir stehst und mich ansiehst", heißt es darin,

"was weißt Du von den Schmerzen, die in mir sind und was weiß ich von den Deinen. Und wenn ich mich vor Dir niederwerfen würde und weinen und erzählen, was wüßtest $\mathrm{Du}$ von mir mehr als von der Hölle, wenn Dir jemand erzählt, sie ist heiß und fürchterlich. Schon darum sollten wir Menschen voreinander so ehrfürchtig, so nachdenklich, so liebend stehn wie vor dem Eingang zur Hölle. « ${ }^{1}$

In diesen wenigen Zeilen formuliert Kafka ein grundlegendes Problem. Man kann nicht sehen, wie es in dem Herzen eines anderen Menschen tatsächlich aussieht. Zwischen den eigenen Schmerzen und den Schmerzen der anderen besteht im Hinblick auf ihre Erkennbarkeit ein Abgrund, den auch die Sprache nur notdürftig zu schließen vermag. Die Fragen, die Kafka stellt, sind genuin epistemischer Art: Was kann ich von den Schmerzen des anderen wissen, wie sein Leid erkennen, wenn weder der Blick von außen noch das gesprochene Wort in der Lage sind, mir eine angemessene Vorstellung von dem zu vermitteln, was in ihm vorgeht? Mit diesen Fragen, die auf die epistemische Dimension der Verletzlichkeit verweisen, möchte ich mich auf den folgenden Seiten beschäftigen.

Wer nicht gesehen wird, wer unsichtbar und infolgedessen auch unerkannt bleibt, der kann auch nicht anerkannt werden. Jeder Akt der Anerkennung setzt einen Akt des Erkennens voraus. Dieser Gedanke, der unter anderem von Paul Ricœur ${ }^{2}$ formuliert wurde, lässt sich von der Frage der Anerkennung individueller und kollektiver

1 Franz Kafka, Briefe 1900-1912 (Kritische Ausgabe), hg. v. Hans-Gerd Koch, Frankfurt a.M. 1999, 28.

2 Vgl. Paul Ricœur, Wege der Anerkennung. Erkennen, Wiedererkennen, Anerkanntsein [2004], übers. v. Ulrike Bokelmann u. Barabara Heber-Schärer, Frankfurt a.M. 2006; ähnlich auch Thomas Bedorf in Verkennende Anerkennung. Über Identität und Politik, Berlin 2010. 
Identitäten auf den Bereich der Verletzlichkeit übertragen. Eine Wunde, die gesehen wird, kann auch versorgt werden. Ihre Sichtbarkeit stellt zwar noch keinen Garanten, wohl aber eine notwendige Voraussetzung dafür dar, dass sich jemand des Leidenden annimmt. Was aber, wenn ein Leiden von niemandem gesehen wird? Wo die Bereitschaft oder die Fähigkeit fehlt, die Verwundung des anderen zu sehen, da kann auch nichts unternommen werden, um seinen Schmerz zu lindern. Besonders virulent wird dieser Zusammenhang dort, wo wir es mit Verletzungen zu tun bekommen, die nicht bloß das Ergebnis eines Unglücks oder Missgeschicks sind, sondern die Folge eines erlittenen Unrechts. Viele Menschen, denen etwas Schlimmes angetan wurde, haben ein Verlangen danach, dass das ihnen zugefügte Unrecht öffentlich anerkannt und die dafür Verantwortlichen klar benannt werden. Dies setzt jedoch voraus, dass der damit verbundene Schmerz erkannt und richtig gesehen wird. Wie ich im Folgenden zeigen möchte, gehören zu den Hindernissen, die sich diesem Erkennen entgegenstellen, nicht nur Gründe externer Art (etwa der Versuch der Verantwortlichen, das von ihnen begangene Unrecht kleinzureden oder zu vertuschen, dem aufseiten der Öffentlichkeit nicht selten die Neigung entgegenkommt, von den Leiden der anderen, insbesondere wenn es sich um Mitglieder einer Minorität handelt, aus Bequemlichkeit keine Notiz zu nehmen), sondern auch solche, die in der merkwürdigen Natur der Schmerzempfindung selber liegen. Der ethischen Frage, wie wir mit dem Faktum menschlicher Verletzlichkeit umgehen sollen, ist also eine epistemische Frage vorgeordnet, die als solche jedoch nur selten explizit wird. Dies ist der Gedanke, den ich auf den folgenden Seiten darlegen möchte.

Hierzu werde ich im ersten Abschnitt zunächst eine Reihe von Überlegungen vorstellen, die die Verletzlichkeit im Allgemeinen betreffen. Im zweiten Abschnitt werde ich dann direkt auf die epistemische Dimension der Verletzlichkeit eingehen. Im dritten und letzten Abschnitt schließlich werde ich zeigen, welche moralischen und politischen Implikationen daraus folgen und inwiefern eine Ethik derVerletzlichkeit von einer Reflexion auf deren epistemische Grundlagen zu profitieren vermag. 
I.

Menschen sind verletzliche Wesen. Sie sind fähig, Verletzungen zuzufügen, und sie sind fähig, Verletzungen zu erleiden. Die Fähigkeit zu handeln, das heißt etwas zu tun, impliziert die Möglichkeit zu verletzen, das heißt jemandem etwas anzutun. Handlungsvermögen (agency) und Verletzlichkeit gehören zusammen. Im Altgriechischen und der Sprache der Lateiner war dieser Zusammenhang noch deutlich gegenwärtig. Wir haben uns daran gewöhnt, das begriffliche Gegenstück zur >Aktion in der >Reaktion`zu erkennen. Der Gegenbegriff zum lateinischen actio lautete ursprünglich jedoch nicht reactio, sondern passio; dem entsprach im Griechischen das Wortpaar

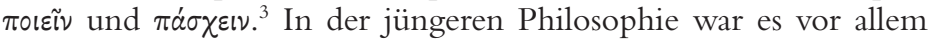
Paul Ricœur, der auf die Notwendigkeit hingewiesen hat, die Theorie des Handelns durch eine des Erleidens zu ergänzen. In einem zuerst 1990 veröffentlichten Aufsatz führt Ricœur aus,

"daß Handeln für einen Handelnden bedeutet, Macht über einen anderen Handelnden auszuüben. Genauer gesagt läßt diese Beziehung, die durch den Ausdruck Macht über ausgedrückt wird, einen Handelnden und einen Erleidenden in Erscheinung treten. [...] Die Handlung wird von jemand anderem erlitten. $\mathrm{Zu}$ dieser grundlegenden Asymmetrie der Handlung kommen all die Perversionen des Handelns hinzu, die darin kulminieren, daß jemand zum Opfer wird:Von der Lüge und List bis hin zur physischen Gewalt und Folter richtet sich die Gewalt unter den Menschen ein als das grundlegende Übel, das im Hintergrund in die asymmetrische Beziehung zwischen Handelndem und Erleidendem inskribiert ist. $\ll^{4}$

Der Punkt, an dem sich Handeln und Erleiden überkreuzen, markiert die Einfallstelle für die Gewalt. Er bildet zugleich ein Kriterium, das es uns ermöglicht, aus den zahlreichen Verwendungsweisen, die das Wort `Verletzung in der Alltagssprache findet, einen bestimmten Bereich auszusondern. Gewiss kann eine Verletzung auch von einem herabstürzenden Ast verursacht werden oder davon, dass ich mich versehentlich an etwas stoße. Moralisch und politisch relevant sind jedoch in erster Linie diejenigen Verletzungen, die

3 Vgl. hierzu Jean Starobinski, Aktion und Reaktion. Leben und Abenteuer eines Begriffspaars [1999], übers. v. Horst Günther, Frankfurt a.M. 2003, 20.

4 Paul Ricœur, Annäherungen an die Person [1990], in: ders., Vom Text zur Person. Hermeneutische Aufsätze (1970-1999), übers. u. hg. v. Peter Welsen, Hamburg 2005, 227-249, hier 243. 
sich einem verantwortungsfähigen Subjekt als Urheber zuschreiben lassen. Und ebendiese Verletzungen sind es, die am Schnittpunkt von Handeln und Erleiden auftauchen. Ob ich nun (mit Ricœur) sage, eine Handlung wird von jemand anderem erlitten, oder ob ich sage, jemand tut jemand anderem etwas an, meint faktisch dasselbe. Die Grundstruktur dieser Art von Verletzungen lässt sich demnach in die Formel bringen: Jemand tut jemandem etwas an. ${ }^{5}$

Dabei muss keineswegs notwendig eine böse Absicht im Spiel sein. Vermutlich hat Hannah Arendt recht, wenn sie konstatiert, dass es selten vorkommt, dass »jemand direkt das Böse will«". Arendt zufolge sind ein Großteil der Verfehlungen, die wir uns Tag für Tag zuschulden kommen lassen, "alltägliche Vorkommnisse, die sich aus der Natur des Handelns selbst ergeben " 7 ; jede Handlung zieht unzählige weitere Handlungen nach sich, so dass niemand in der Lage ist, die Konsequenzen seines Tuns (und Unterlassens) komplett zu überschauen und zu antizipieren, wo diese potenziell verletzend sein können. Dieser Umstand hat Arendt jedoch nicht daran gehindert, immer wieder darauf hinzuweisen, dass gerade in der Gedankenlosigkeit, mit der viele Dinge getan werden, eine unversiegbare Quelle des Übels liegt. ${ }^{8}$ Ethik ist nicht zuletzt auch eine Frage der Aufmerksamkeit.

Während das Handlungsvermögen in das Register derjenigen Fähigkeiten gehört, die das Porträt des "fähigen Menschen «" ausmachen, gehört dieVerletzlichkeit in das Register derjenigen Vermögen, die aus der menschlichen Affektivität resultieren. Zwar trifft es zu, dass sich die "Chiffren der Verletzlichkeit ${ }^{10}$ von Kultur zu Kultur und von Epoche zu Epoche unterscheiden (Ehrverletzungen, um ein augenfälliges Beispiel zu nennen, spielen in unserer Gesellschaft nicht mehr dieselbe Rolle wie noch im Preußen des 19. Jahrhun-

\footnotetext{
5 Vgl. Bernhard Waldenfels, Aporien der Gewalt, in: Gewalt. Strukturen, Formen, Repräsentationen, hg. v. Mihran Dabag u.a., München 2000, 9-24, hier 13.

${ }^{6}$ Hannah Arendt, Vita activa oderVom tätigen Leben [1958], München ${ }^{7} 2008,306$.

7 Ebd.

${ }^{8}$ Vgl. Hannah Arendt, Eichmann in Jerusalem. Ein Bericht von der Banalität des Bösen [1964], übers. v. Brigitte Granzow, München ${ }^{15} 2006$.

9 Der »fähige Mensch" (homme capable) bildet das emblematische Zentrum der phänomenologischen Anthropologie Paul Ricœurs; vgl. ders., Das Selbst als ein Anderer [1990], übers. v. Jean Greisch in Zusammenarbeit mit Thomas Bedorf u. Birgit Schaaff, München ${ }^{2} 2005$.

${ }^{10}$ So der Untertitel der deutschen Übersetzung eines Buches von Elaine Scarry, auf das ich weiter unten noch zu sprechen kommen werde.
} 
derts $^{11}$ ); allen Verletzungen ist jedoch gemeinsam, dass sie sich als ein negatives Affizierungsgeschehen charakterisieren lassen. Die affektive Dimension der Verletzlichkeit zeigt sich unter anderem daran, wie uns Verletzungen primär gegeben sind: Wenn wir verletzt werden, dann fühlen wir das - und zwar als Schmerz. Ein Schlag ins Gesicht oder ein beleidigendes Wort werden nicht bloß sachlich registriert, sondern unmittelbar und direkt als etwas empfunden, das weh tut. Weit davon entfernt, ein bloßer Akt der Wahrnehmung oder der Kognition zu sein, ist uns dieVerletzung unmittelbar als Schmerz gegeben. Der Schmerz bildet den affektiven Kern der Erfahrung der Verletzlichkeit; er ist gewissermaßen der Rohstoff, aus dem sämtliche Verletzungen fabriziert sind.

Jede Verletzung lässt sich gleichsam von zwei Seiten her in den Blick nehmen, von außen und von innen: Die Außenseite, das ist die Handlung, das Geschehen oder Ereignis, das die Verletzung verursacht. Dieses Geschehen kann äußerst subtil und unscheinbar sein und von niemandem, außer dem Verletzten selbst, bemerkt werden. Gleichwohl handelt es sich dabei jedoch um etwas, das sdraußen in der Welt passiert. Von ihrer Außenseite her betrachtet, besitzen Verletzungen also den gegenständlichen Charakter eines Sachverhalts, der, als Teil der empirischen Realität, grundsätzlich auch von anderen gesehen und erkannt werden kann. Mit dem Schmerz hingegen verhält es sich anders. Er bildet die affektive Innenseite derVerletzung. Absolute Evidenz und den Charakter einer primären Realität besitzt der Schmerz nun aber nur für denjenigen, der selber von ihm betroffen ist. Sobald man den Fokus der Reflexion auf die affektive Dimension der Verletzlichkeit verlagert, bekommt man es also mit einem Erkenntnisproblem zu tun, das sich in der Frage verdichtet, wie man als Außenstehender zu dieser Ebene überhaupt Zugang gewinnen kann.

II.

In epistemischer Hinsicht sind Schmerzen merkwürdig fragil. Diese Fragilität betrifft jedoch nicht die Erkennbarkeit der eigenen Schmerzen, sondern ausschließlich die der anderen. Erinnern wir uns an das Kafka-Wort: Selbst dann, wenn sich jemand vor mir niederwerfen und mir unter Tränen von seinem Schmerz erzählen

${ }^{11}$ Vgl. dazu etwa Ludgera Vogt/Arnold Zingerle, Zur Aktualität des Themas Ehre und zu seinem Stellenwert in der Theorie, in: Ehre. Archaische Momente in der Moderne, hg. v. dens., Frankfurt a.M. 1994, 9-34. 
würde, wüsste ich von ihm und davon, wie es um seine Verfassung tatsächlich bestellt ist, auch nicht viel mehr als jemand von der Hölle weiß, dem mitgeteilt wird, es sei dort heiß und fürchterlich. Man kann das Problem natürlich auch nüchterner ausdrücken. So heißt es bei Ludwig Wittgenstein in den Philosophischen Untersuchungen lakonisch: "Das ist richtig: es hat Sinn, von Andern zu sagen, sie seien im Zweifel darüber, ob ich Schmerzen habe; aber nicht, es von mir selbst zu sagen. ${ }^{12}$ Sowohl bei Kafka als auch bei Wittgenstein kündigt sich die Frage nach der epistemischen Dimension der Verletzlichkeit also als das Problem eines den Schmerz betreffenden Evidenzgefälles an.

Die Feststellung, dass zwischen den eigenen Schmerzen und den Schmerzen der anderen ein fundamentaler Unterschied besteht, bildet auch den Ausgangspunkt der 1985 erschienenen Monographie The Body in Pain von Elaine Scarry, einem Buch, das in meinen Augen nach wie vor zu den maßgeblichen Arbeiten gehört, die zu diesem Thema geschrieben wurden. ${ }^{13}$ In einer längeren Passage buchstabiert Scarry darin aus, was es mit diesem Evidenzgefälle für eine genaue Bewandtnis hat:

"Spricht man über `die eigenen körperlichen Schmerzen und über sdie körperlichen Schmerzen der anderen`, so hat es bisweilen den Anschein, als spräche man von zwei gänzlich verschiedenen Dingen. Jemand, der von Schmerzen heimgesucht wird, nimmt den Schmerz 'mühelos wahr, ja, er kann es gar nicht vermeiden, ihn wahrzunehmen; für die anderen dagegen ist 'mühelos gerade, ihn nicht wahrzunehmen (es ist leicht, den Schmerz des anderen zu übersehen; selbst wenn man sich nach Kräften bemüht, mögen Zweifel bleiben, ob er wirklich da ist, und es bleibt auch die verblüffende Freiheit, seine Existenz zu leugnen; wenn man ihn jedoch unter Aufbietung aller Aufmerksamkeit wahrnimmt, dann ist, was man da wahrnimmt, in seiner Unannehmlichkeit nur ein Schatten dessen, was der wirkliche Schmerz ist). Für einen Menschen, der Schmerzen hat, ist der Schmerz fraglos und unbestreitbar gegenwärtig, so daß man sagen kann, Schmerzen zu haben sei das plausibelste Indiz dafür,

\footnotetext{
${ }^{12}$ Ludwig Wittgenstein, Philosophische Untersuchungen [1953], auf der Grundlage der Kritisch-genetischen Edition neu hg. v. Joachim Schulte, Frankfurt a.M. 2003, 147.

${ }_{13}$ Vgl. Elaine Scarry, The Body in Pain. The Making and the Unmaking of the World, New York 1985; dt. unter dem Titel Der Körper im Schmerz. Die Chiffren der Verletzlichkeit und die Erfindung der Kultur, übers. v. Michael Bischoff, Frankfurt a.M. 1992.
} 
was es heißt, `Gewißheit zu haben dieselbe Erfahrung so schwer faßbar, daß ıvon Schmerzen hören als Paradebeispiel für Zweifeln gelten kann. So präsentiert der Schmerz sich uns als etwas Nichtkommunizierbares, das einerseits nicht zu leugnen, andererseits nicht zu beweisen ist. ${ }^{14}$

Der Schmerz besitzt in epistemischer Hinsicht eine eigentümliche Doppelnatur, die sich entlang der Pole rabsolut evident bezweifelbar organisiert.

Wenn Scarry die Vermutung ausspricht, dass »der Schmerz wohl auch deshalb so widerwärtig [ist], weil er zwischen der eigenen Realitätswahrnehmung und der Realität der anderen eine unüberwindbare Mauer errichtet ${ }^{15}$, dann deutet sich darin bereits an, inwiefern das epistemologische Thema des Erkennens und das ethische Thema der Anerkennung zusammenhängen. So wie die vertikale Asymmetrie zwischen den Polen Handeln und Erleiden die Einlassstelle bildet für die Gewalt, die verletzt, so bildet die Asymmetrie zwischen den Polen `Gewissheit und `Zweifek die Einlassstelle für einen Verdacht, der mitunter so stark sein kann, dass er das Vertrauen in die fiduziarische Kraft der Sprache komplett unterminiert. Der Schmerz widersetzt sich der Sprache; in extremen Fällen geht er sogar so weit, sie ganz zu zerstören. ${ }^{16}$ Wer dennoch versucht, seinen Schmerz zum Ausdruck zu bringen, läuft stets Gefahr, dass man seinen Worten keinen Glauben schenkt. Wie oft haben wir nicht schon, nachdem uns jemand von seinem Leid erzählt hat, gedacht, der übertreibt doch, so schlimm kann das Ganze nicht sein? Manchmal gibt es gute Gründe, die dieses Misstrauen rechtfertigen. In denjenigen Fällen, in denen es unbegründet ist, stellt der Akt des Zweifelns jedoch selbst eine Art epistemische Verletzung dar: Es tut weh, wenn einem die anderen nicht glauben. DerVerdacht ruft einen zzweiten< Schmerz hervor, der zu dem bereits gegebenen Schmerz noch verstärkend hinzukommt. Die Mauer der Ungewissheit, die

\footnotetext{
${ }^{14}$ Scarry, Der Körper im Schmerz, 12.

15 Ebd. - Dies gilt im Übrigen nicht nur für starke Schmerzen, sondern bereits für ein vergleichsweise so harmloses Leiden wie Heuschnupfen: Die Welt des Allergikers ist im Frühsommer definitiv eine andere als die des Nicht-Allergikers.

${ }_{16}$ Wer starke Schmerzen hat, ist häufig kaum noch in der Lage, einen vollständigen Satz zu sprechen; an die Stelle der zusammenhängenden Rede treten einzelne, unter Mühe herausgepresste Wörter. Wird der Schmerz noch stärker, so löst er nicht nur die Syntax, sondern auch die phonetische und semantische Struktur der Sprache auf; die Wörter werden abgelöst von einem bloßen Schreien, Stöhnen, Keuchen, Ächzen. Die Klage, an der Grenze von Sprache und Nicht-Sprache situiert, nimmt hier die Gestalt von Lauten an, die auch die eines Tieres sein könnten, Laute, die nicht mehr aus der Person, sondern nur noch aus ihrem Körper herauszukommen scheinen.
} 
der Schmerz zwischen den Subjekten etabliert, bewirkt also »eine Verdoppelung der auflösenden Kraft des Schmerzes; die mangelnde Erkennbarkeit und Anerkennung [...] werden zu einer zweiten Form der Negation und Abweisung, zum sozialen Äquivalent der physischen Widerwärtigkeit « ${ }^{17}$.

Warum aber ist das so? Woher rührt die Schwierigkeit, über den Schmerz zu sprechen? Über die Schmerzen der anderen können wir wenig sagen, weil wir zu ihnen keinen unmittelbaren Zugang haben. Wie lässt sich aber erklären, dass wir auch den eigenen Schmerz nur so schwer zur Sprache bringen können, wenn es sich dabei doch um etwas handelt, das uns ganz eindeutig und ohne jeden Zweifel gegeben ist?

Einer Antwort auf diese Fragen kommt man näher, wenn man sich vor Augen hält, dass der Schmerz keinen anderen Gehalt hat als den seiner "schiere[n] Widerwärtigkeit « ${ }^{18}$. Was einen Schmerz zum Schmerz macht (und woran man ihn erkennt), ist nichts als das fait brut, dass er weh tut. Hören wir dazu noch einmal Elaine Scarry:

"Während andere Empfindungen einen Inhalt haben, der positiv, neutral oder negativ sein mag, ist der Inhalt von Schmerz die Negation schlechthin. Wenn ein Mensch die eigenen Schmerzen nicht als unangenehm empfindet, wenn seine Schmerzen keine Abscheu in ihm erwecken, dann können sie weder nach philosophischem Verständnis noch nach psychologischer Definition als Schmerzen bezeichnet werden. Schmerz ist die reine physische Erfahrung der Negation, [...] die Wahrnehmung von etwas, das gegen uns ist, und von etwas, gegen das man sein muß. $\aleph^{19}$

Der Schmerz hat keine intentionale Struktur. Ihm entspricht kein Objekt in der Welt. Daraus resultiert die Schwierigkeit, den Schmerz zur Sprache zu bringen.

"Obwohl die Fähigkeit, körperlichen Schmerz zu empfinden, zu unseren ureigensten Fähigkeiten gehört, geradeso wie die Fähigkeit, zu hören, zu fühlen, Wünsche, Angst oder Hunger zu haben, unterscheidet sie sich davon und von allen sonstigen körperlichen und psychischen Erscheinungen dadurch, daß sie kein Objekt in der äußeren Welt hat. Wenn wir hören oder fühlen, so hören oder fühlen wir etwas, das außerhalb des Körpers liegt; ein Wunsch ist ein Wunsch nach $\mathrm{x}$, Angst ist Angst vor $\mathrm{y}$, Hunger ist

\footnotetext{
17 Scarry, Der Körper im Schmerz, 85.

${ }^{18}$ Ebd., 79.

${ }^{19}$ Ebd.
} 
Hunger nach z; doch Schmerz ist nicht Schmerz von oder nach etwas; Schmerz ist nur er selbst. Diese Objektlosigkeit, das Fehlen jeglichen referentiellen Gehalts, macht es nahezu unmöglich, ihn in Worte zu fassen. ${ }^{20}$

Der Schmerz verweist auf nichts anderes als sich selbst. Nicht nur, dass er selbst kein Objekt hat, besitzt er zugleich die Tendenz, jedes andere Objekt aus unserem Bewusstsein zu verdrängen. Im Extremfall betrifft seine zersetzende Wirkung nicht nur die Fähigkeit zu sprechen, sondern sämtliche perzeptiven, kognitiven und affektiven Vermögen:Wer starke Schmerzen hat, büßt die Fähigkeit ein, die Welt um sich herum richtig wahrzunehmen und sich zu ihr in Beziehung zu setzen; ab einem bestimmten Punkt ist er nicht mehr in der Lage, an irgendetwas anderes zu denken oder irgendetwas anderes zu fühlen. Alles, was sein Dasein ausmacht, wird nur noch von dieser einen Sache bestimmt. Wie die meisten Regime, so neigt auch dasjenige, das der Schmerz über das Bewusstsein und den Körper errichtet, zur Totalisierung. Wenn sich der Schmerz der Sprache widersetzt, dann also auch deshalb, weil er der Welt als Ganzes entgegensteht, weil er dazu neigt, die Fülle der Dinge und Beziehungen, die die Welt eines Menschen ausmachen, aufzulösen und sich an deren Stelle zu setzen.

In dem $\mathrm{Maße}$, in dem der Schmerz seine rentweltlichender Wirkung entfaltet, in dem Maße schließt er den Leidenden in sich ein. Seine Widerwärtigkeit rührt nicht zuletzt daher, dass er in mir ist und dass er die totalisierende Tendenz besitzt, dieses >Innen plett auszufüllen. Der Schmerz besitzt einen conatus ganz eigener Art: Er strebt danach, sich in seinem Sein zu erhalten und noch zu steigern. Der Schmerz will wachsen. Er will aufs Ganze. Bis zu dem Punkt, an dem sich mein gesamter Körper in einen Kriegsschauplatz verwandelt hat.

Scarry geht es in ihrer Untersuchung ausdrücklich um das Phänomen körperlicher Schmerzen. Was sie über die epistemische Unzugänglichkeit der Schmerzen der anderen sagt, lässt sich von dem Bereich somatischer Leiden und physischer Verletzungen aber auch auf den Bereich derjenigen Verletzungen übertragen, von denen primär nicht der Körper betroffen ist, sondern die Psyche. Der zentrale Unterschied zwischen körperlichen und psychischen Schmerzen besteht darin, dass erstere keine intentionale Struktur aufweisen, also nicht auf ein Objekt oder einen Sachverhalt in der Welt bezogen sind, letztere hingegen schon. Diese Differenz verflüchtigt sich jedoch, sobald es nicht mehr nur um den Schmerz an

${ }^{20}$ Ebd., 242. 
sich geht, als isolierte Empfindung, sondern um das umfassendere Affizierungsgeschehen, in das er jeweils eingebettet ist.

Richtet man sein Augenmerk nicht allein auf die Empfindung des Schmerzes, sondern auf die konkreten Verletzungen, die ihn verursacht haben, dann wird deutlich, dass eine klare Trennlinie zwischen sphysisch und spsychisch so einfach nicht zu ziehen ist. So etwas wie eine srein physischer Gewalt - das heißt eine Gewalt, die nur den Körper verletzt und die Psyche darüber vollkommen unangetastet lässt - gibt es vermutlich nur als Grenzfall. Das Paradigma für die Zufügung körperlicher Schmerzen erkennt Scarry im Krieg und in der Folter. ${ }^{21}$ Dass der körperliche Schmerz im Zentrum der Folter steht, heißt jedoch nicht, dass jemand, der gefoltert wird, nicht auch an seiner Seele Schaden nimmt. Dasselbe gilt für jemanden, der äußerlich unversehrt aus einem Krieg zurückkehrt (ich werde im nächsten Abschnitt darauf zurückkommen). Umgekehrt gilt, dass auch die meisten symbolischen Verletzungen sich früher oder später auf physiologische oder somatische Weise bemerkbar machen: Wer jahrelang gemobbt wird, bekommt irgendwann vermutlich Bauchschmerzen; einem gläubigen Christen kann sich beim Anblick eines umgedrehten Kreuzes buchstäblich der Magen umdrehen; Angst ist ein viszerales Gefühl.

Ein Schmerz, der auf eine psychische Verletzung zurückgeht, hört allein deshalb, dass er sich mit einem intentionalen Gehalt verbindet, nicht auf, Schmerz zu sein. Ebenso wie körperliche Schmerzen können auch psychische Leiden - man denke etwa an Depressionen eine Tendenz zur Totalisierung entwickeln, die sich mit dem Gefühl verbindet, von der Welt und allen anderen Menschen abgeschnitten $\mathrm{zu}$ sein. Kurzum, auch wenn es zutrifft, dass zwischen physischen und psychischen Schmerzen ein Unterschied besteht, so ist dieser Unterschied doch nicht so groß, dass er beide in vollkommen getrennte Register verweisen würde.

Wenn es primär um die Erkennbarkeit geht, dann verhält es sich so, dass psychische Verletzungen tendenziell sogar noch schwieriger zu erkennen sind als körperliche. Physische Gewalt hinterlässt meist Spuren auf dem Körper, über den sie hinweggeht. Diese Spuren sind sichtbar. Sie können von anderen gelesen werden. ${ }^{22}$ Bei Akten see-

${ }^{21}$ Vgl. ebd., 43-90 (I. Kapitel: Die Struktur der Folter), 91-237 (II. Kapitel: Die Struktur des Krieges).

${ }^{22}$ Manchmal, wenn das Individuum selber dazu nicht mehr in der Lage ist, sind es gleichsam die Wunden, die an seiner Stelle das Wort ergreifen und dem Mediziner oder den Umstehenden ssagen`, was zu tun ist: die Beule 'will gekühlt werden, die Schnittwunde 'verlangt danach, dass man sie verbindet und die Blutung stoppt etc. Lesbar sind diese Spuren aber nicht nur im Hinblick auf die jetzt in diesem Moment 
lischer Gewalt ist das nicht in derselben Weise möglich. Der Anblick eines zerschmetterten Kiefers vermittelt zwar auch keinen direkten Zugang zu der Realität des Schmerzes, aber er besitzt doch eine Form der Evidenz, die Sichtbarkeit einer empirischen Realität, die psychischen Verletzungen abgeht. Von seiner Mutter im Stich gelassen oder gedemütigt zu werden, kann ebenso wehtun wie von seinem Vater verprügelt zu werden. Ein Kind, das zu Hause regelmäßig körperliche Misshandlungen erfährt, wird in unserer Gesellschaft jedoch eher damit rechnen können, dass jemand von seinem Leid Notiz nimmt, als eines, das zu Hause subtileren Formen der Gewalt ausgesetzt ist.

Epistemisch fragil ist der Schmerz auch insofern, als es kein $\mathrm{Maß}$ oder Verfahren gibt, das es erlauben würde, allgemein verbindliche Aussagen darüber zu machen, wie sehr etwas weh tut. "Ein Individuum", schreibt Max Scheler, "kann an demselben Grade eines Schmerzes mehr oder weniger leiden als ein anderes Individuum. $\mathbb{1}^{23}$ Dies gilt, wie wissenschaftliche Experimente gezeigt haben, bereits für die Empfindung von körperlichen Schmerzreizen. ${ }^{24}$ Dass alle Menschen verletzlich sind, heißt nicht, dass ein und dieselbe Handlung von allen in derselben Weise oder im selben Maße als verletzend empfunden wird. Das allgemeine Faktum derVerletzlichkeit inkarniert sich individuell verschieden; in jeder menschlichen Existenz sind die swunden Punkter ein wenig anders verteilt. Ein bestimmtes Schimpfwort, das den einen kalt lässt, mag den anderen aufs Empfindlichste treffen, eben weil es an einen solchen Punkt rührt. Umgekehrt wird dieser eine bestimmte Geste als ungemein kränkend empfinden, von der jener vollkommen unberührt bleibt. Die Empfindung des Schmerzes lässt sich nur bedingt objektivieren. Dies ist der Grund, weshalb man davon sprechen kann, dass jede Verletzung in affektiver Hinsicht - das heißt im Hinblick auf den mit ihr verbundenen Schmerz - singulär und unvergleichlich ist. Der Schmerz existiert gleichsam nur im Singular, als unvermeidlich Partikulares, und paradoxerweise ist es gerade diese Singularität, die ihm seine potenzielle Maßlosigkeit verleiht. Das ist die Hölle, von

und in der weiteren Zukunft zu ergreifenden therapeutischen Maßnahmen, sondern auch im Hinblick darauf, was in der Vergangenheit geschehen ist und zu der Verletzung geführt hat. Die moderne Forensik hat eine ganz erstaunliche Kunstfertigkeit darin entwickelt, aus der Art der Zerstörungen, die ein Körper aufweist, Rückschlüsse auf die Ursache dieser Zerstörungen zu ziehen.

${ }^{23}$ Max Scheler, Der Formalismus in der Ethik und die materiale Wertethik. Neuer Versuch der Grundlegung eines ethischen Personalismus [1913], Bern ${ }^{4} 1954,263$.

${ }^{24}$ Vgl. Joachim Bauer, Schmerzgrenze. Vom Ursprung alltäglicher und globaler Gewalt, München 2011, 68. 
der Kafka spricht. Wir können nicht wissen, wie es dort aussieht, und die, die es wissen, sind wie durch eine unsichtbare Mauer von allen anderen getrennt. Der epistemische Abgrund bleibt.

III.

Verletzungen begründen Ansprüche. Dies gilt in den Sphären des Rechts und der Politik ebenso wie im moralischen Miteinander des alltäglichen Umgangs. Wer beleidigt wurde, darf erwarten, dass man sich bei ihm entschuldigt, wer Teil einer Gruppe ist, die systematisch diskriminiert wird, hat ein Anrecht darauf, dass diese Diskriminierung ein Ende findet, wer körperlich versehrt oder wem etwas Wertvolles genommen wurde, hat einen Anspruch auf Schmerzensgeld oder Schadenersatz. In der Praxis sind diese Forderungen natürlich meistens stark umstritten und Gegenstand von Auseinandersetzungen, die außerordentlich komplex sein können. Der epistemischen Dimension der Verletzlichkeit kommt dabei jedoch stets eine entscheidende Rolle zu. Denn geltend machen lassen sich diese Ansprüche nur, wenn es gelingt, die erlittene Verletzung auch intelligibel zu machen. ${ }^{25}$

Ein anschauliches Beispiel dafür findet sich in Michael Sandels Buch Gerechtigkeit. ${ }^{26}$ In den USA wurde vor einigen Jahren eine Debatte darüber geführt, wer das Purple Heart verdient, einen militärischen Orden, der an Soldaten verliehen wird, die im Kampfeinsatz verwundet oder getötet wurden. »Im Gegensatz zu anderen militärischen Auszeichnungen", so unterrichtet uns Sandel, "wird mit dem Purple Heart nicht Tapferkeit honoriert, sondern das Opfer, das jemand gebracht hat. Dazu ist kein heroischer Akt erforderlich, nur eine durch den Feind zugefügte Verwundung. ${ }^{27}$ Bei vielen amerikanischen Soldaten, die aus den Kriegen in Afghanistan und dem Irak zurückkehrten, wurde eine >Posttraumatische Belastungsstörung diagnostiziert, zu deren Symptomen wiederkehrende Alpträume, schwere Depressionen und eine hohe Suizidgefahr

\footnotetext{
${ }^{25}$ Dieser Zusammenhang überschneidet sich - was hier nicht näher ausgeführt werden kann - mit Judith Butlers Begriffen der Betrauerbarkeit (grievability) und Prekarität (precariousness) sowie mit den Zusammenhängen, die Miranda Fricker in ihrem gleichnamigen Buch unter dem Titel epistemic injustice diskutiert; vgl. Judith Butler, Frames of War. When Is Life Grievable? London/New York 2009; Miranda Fricker, Epistemic Injustice. Power and the Ethics of Knowing, Oxford/New York 2007.

${ }^{26}$ Vgl. Michael J. Sandel, Gerechtigkeit. Wie wir das Richtige tun [2009], übers. v. Helmut Reuter, Berlin 2013, 18-21.

${ }^{27}$ Ebd., 19.
} 
gehören. Fürsprecher dieser Veteranen machten den Vorschlag, auch diese Soldaten mit dem Purple Heart zu ehren, »da psychische Verletzungen mindestens ebenso quälend sein können wie körperliche Wunden. ${ }^{28}$ Diese Forderung wurde 2009 vom Pentagon abgelehnt. Zwar wurde beschlossen, den Veteranen, die an psychischen Traumata litten, dieselbe medizinische Unterstützung zuteilwerden zu lassen wie denjenigen Soldaten, die physisch verwundet worden waren. Die militärische Ehrung blieb ihnen jedoch vorenthalten. Als Gründe für diese Entscheidung nannte das Pentagon zum einen, dass Posttraumatische Belastungsstörungen "nicht durch absichtliche Feindeinwirkung verursacht" würden, und zum anderen, dass sie "objektiv nur schwer zu diagnostizieren ${ }^{29}$ seien.

Auch wenn der Initiative der Soldaten kein Erfolg beschieden war, so konnten sie sich in ihrem Kampf um Anerkennung doch zumindest auf ein bestimmtes medizinisch-psychologisches Wissen berufen, das ihrem Leiden einen Namen verliehen hat: Posttraumatische Belastungsstörung. Ungleich schwieriger fällt der Kampf um die Anerkennung des eigenen Leids dort aus, wo es einen solchen $\mathrm{Na}-$ men noch gar nicht gibt, wo die Sprache (der Medizin, des Rechts, der Politik, der Psychologie, der Moral) keine Ausdrücke, Konzepte und Kategorien bereitstellt, die es einem ermöglichen, sein Leiden in einer Weise zu artikulieren, die verstanden wird. ${ }^{30}$ Das epistemische Problem der mangelnden Intelligibilität des Schmerzes und das moralische und politische Problem der Anerkennung bestimmter Formen von Leid und Unrecht hängen also eng zusammen.

Es ist nicht davon auszugehen, dass in den Führungsstäben des amerikanischen Militärs sonderlich viel auf die Literatur gegeben wird (vor allem nicht auf eine, die so enigmatisch daherkommt wie diejenige Kafkas). Gleichwohl darf, versuchshalber, die Frage erlaubt sein, ob die Entscheidung des Pentagons genauso ausgefallen wäre, wenn sich die Verantwortlichen an dem orientiert hätten, was in Kafkas Brief an Oskar Pollak als ethische Botschaft aufblitzt. Kehren wir daher, zum Abschluss, noch einmal zu den eingangs zitierten Zeilen zurück:

"Wenn Du vor mir stehst und mich ansiehst, was weißt Du von den Schmerzen, die in mir sind und was weiß ich von den Deinen. Und wenn ich mich vor Dir niederwerfen würde und

${ }^{28}$ Ebd., 18.

${ }^{29}$ Ebd., 19.

${ }^{30}$ Miranda Fricker analysiert dies ausführlich (und mit dem dieser Frage eigentlich gebührenden Grad an Komplexität) anhand der Etablierung des Konzepts der sexuellen Belästigung (sexual harrassment); vgl. Fricker, Epistemic Injustice, 147-175. 
weinen und erzählen, was wüßtest Du von mir mehr als von der Hölle, wenn Dir jemand erzählt, sie ist heiß und fürchterlich. Schon darum sollten wir Menschen voreinander so ehrfürchtig, so nachdenklich, so liebend stehn wie vor dem Eingang zur Hölle."

Der literarische Ausdruck (und vor allem der eindrückliche Vergleich am Ende) täuscht leicht darüber hinweg, dass Kafka dem von ihm formulierten Gedanken eine Struktur verliehen hat, die in ihrer formalen Gliederung an einen philosophischen Syllogismus erinnert (freilich ohne senso strictu einer zu sein). Kafka entwickelt sein >Argument<, wenn man so sagen darf, in einem Dreischritt, wobei die ersten beiden Sätze gewissermaßen die Prämissen bilden und der dritte Satz die Konklusion:

1. Wenn Du vor mir stehst und mich ansiehst, was weißt Du von den Schmerzen, die in mir sind und was weiß ich von den Deinen.

2. Und wenn ich mich vor Dir niederwerfen würde und weinen und erzählen, was wüßtest Du von mir mehr als von der Hölle, wenn Dir jemand erzählt, sie ist heiß und fürchterlich.

3. Schon darum sollten wir Menschen voreinander so ehrfürchtig, so nachdenklich, so liebend stehn wie vor dem Eingang zur Hölle.

Die Sätze eins und zwei bilden den eigentlich sepistemologischen Teil des Gedankens. In ihnen wird nach dem gefragt, was wir von den Schmerzen unseres Gegenübers wissen können. Wie es sich für ein ordentliches Stück Erkenntniskritik gehört, wird in den vorangestellten Konditionalsätzen jeweils das spezifische Set an Bedingungen angegeben, unter denen sich diese Frage stellt: Im ersten Satz geht es dabei zunächst um die visuelle Wahrnehmung und um eine Situation, die keiner besonderen Intimität bedarf (voreinander stehen und sich ansehen kann man auch noch auf eine gewisse Entfernung). Im zweiten Satz kommt es dann zu einerVerschärfung und dramatischen Zuspitzung der Frage: Die räumliche Distanz verringert sich, zur visuellen Wahrnehmung hinzu kommt der gestische (sich vor dem anderen niederwerfen), vorsprachliche (weinen) und sprachliche Ausdruck (erzählen), eine ganze Reihe an expressiven Handlungen also, die darauf abzielen, die durch den Schmerz gesetzte Trennung zu überwinden. 
Es ist auffällig, dass Kafka die ersten beiden Sätze zwar als Fragen formuliert, an ihr Ende jedoch kein Fragezeichen gesetzt hat (wie es die Grammatik in diesem Fall eigentlich verlangen würde), sondern einen Punkt - ganz so, als ob das Rhetorische der Fragen, der Umstand, dass die Antwort in beiden Fällen nur negativ und ernüchternd ausfallen kann, durch das Fehlen des Fragezeichens noch zusätzlich unterstrichen werden sollte. Es ist die Position eines strikten erkenntniskritischen Skeptizismus, die hier zum Ausdruck kommt. In der Form der Frage - »Wenn Du vor mir stehst und mich ansiehst, was weißt Du von den Schmerzen, die in mir sind und was weiß ich von den Deinen" - ist die negative Antwort bereits impliziert: Nichts oder so gut wie gar nichts. Punkt. Und auch wenn einer von uns beiden nun anfangen würde $z u$ weinen und zu erzählen, wäre dasjenige, was die Erzählung vermitteln würde, bestenfalls (um nochmal die Formulierung von Elaine Scarry aufzugreifen) "ein Schatten dessen, was der wirkliche Schmerz ist ${ }^{31}$.

Nun bleibt Kafka jedoch nicht dabei stehen, die mangelnde Intelligibilität der Schmerzen des anderen zu konstatieren. Stattdessen formuliert er im darauffolgenden dritten Satz eine Aussage, die unverkennbar einen ethischen Charakter aufweist. Kafka skizziert darin, wie wir "voreinander [...] stehn", das heißt in welcher Haltung wir einander gegenübertreten und begegnen sollten, nämlich nachdenklich, ehrfürchtig und liebend. Der normative Anspruch der Aussage (»darum sollten wir«) wird zusätzlich unterstrichen durch den Wechsel der Personalpronomina:Während in den Sätzen zuvor nur von der ersten und der zweiten Person Singular die Rede war, im raschen reziproken Wechsel, ist nun ein generisches Wir angesprochen ("wir Menschen«). Im Übergang vom zweiten zum dritten Satz kommt es also zu einem Sprung und Wechsel der Ebenen: Auf ein epistemisches Problem, das sich als unlösbar erweist, antwortet Kafka, indem er eine ethische Aussage formuliert.

Das Bemerkenswerte und die eigentliche Pointe des Gedankens liegt in den beiden Wörtern, mit denen Kafka den dritten Satz an das vorher Gesagte anschließt, in der Konjunktion "Schon darum", die den Sprung von der Epistemologie zur Ethik zugleich vollzieht und verschleiert, indem sie dem Schlusssatz den Charakter einer logisch zwingenden Schlussfolgerung verleiht: Nicht obwohl, sondern gerade weil wir von den Schmerzen des anderen nur so wenig wissen können, sind wir dazu angehalten, ihm mit Ehrfurcht, Nachdenklichkeit und Liebe zu begegnen. Die Unlösbarkeit des Problems, wie wir die Schmerzen des anderen zweifelsfrei erkennen können, ent-

${ }^{31}$ Scarry, Der Körper im Schmerz, 12. 
bindet uns nicht von der Frage, wie wir mit diesem anderen umgehen und uns ihm gegenüber verhalten sollen. Und ebendiese Frage ist es, auf die der Schlusssatz eine Antwort gibt. Kafka skizziert in ihm so etwas wie den Kern einer Ethik der Verletzlichkeit, die gerade aus dem Wissen um deren epistemische Opazität erwächst.

Nun wirft diese Antwort jedoch selbst ein neues Rätsel auf: Dass man ehrfürchtig und auch nachdenklich vor dem Eingang zur Hölle steht, mag ja noch angehen - aber liebend? Vielleicht lässt sich der darin verborgene Anspruch etwas depotenzieren, indem man die Trias $>$ Nachdenklichkeit, Ehrfrucht, Liebe durch einen zeitgenössischeren Ausdruck wie >Respekt oder >Achtung ersetzt. Frei übersetzt würde Kafkas Botschaft also lauten: Nichts oder so gut wie gar nichts weiß ich von deinen Schmerzen (und weißt du von meinen). Gerade deshalb aber sollten wir einander mit Achtung und mit Vorsicht begegnen. Das Leiden der anderen verdient Respekt gerade in seiner Unzugänglichkeit.

- Fabian Bernhardt ist Doktorand am Institut für Philosophie der Freien Universität Berlin. Zu seinen Arbeitsgebieten gehören der Zusammenhang von Recht und Emotion sowie Fragen des Umgangs mit Schuld und vergangenem Unrecht. 